\title{
A REPRESENTATION THEORY FOR PRIME AND IMPLICATIVE SEMILATTICES
}

\author{
BY \\ RAYMOND BALBES
}

1. Introduction. A large part of the theory of pseudo complemented lattices can be extended to pseudo complemented semilattices, as was pointed out by O. Frink in [3]. In [5], W. Nemitz makes a similar observation concerning implicative lattices and implicative semilattices. In the first part of this paper we find necessary and sufficient conditions for a semilattice $L$ to be isomorphic with a family $R$ of sets so that finite products in $L$ correspond to intersections in $R$, and finite sums in $L$-when they exist-correspond to unions in $R$. Semilattices satisfying this condition will be called prime. The term isomorphism is used for a correspondence between partially ordered sets which preserves order in both directions [1, p. 3].

In $\S 3$, a representation theory for prime semilattices is presented. It shows that a prime semilattice $L$ is essentially a collection $C$ of compact-open sets of a Stone space. As expected, $L$ is a lattice if and only if $C$ consists of all compact-open sets. Thus, we have a natural generalization of the Stone representation theorem for distributive lattices [8]. An interesting example of a prime semilattice is an implicative semilattice. The last section is concerned with the properties of the representation space of these semilattices.

2. Prime semilattices. For a subset $\left\{x_{1}, \ldots, x_{n}\right\}$ of a semilattice $L, x_{1} \cdots x_{n}$ will denote the greatest lower bound; the least upper bound-if it exists-will be denoted by $x_{1}+\cdots+x_{n}$.

Definition 2.1. A nonempty subset $F$ of a semilattice is a filter in $L$ provided:

(1) If $x \in F$ and $x \leqq y$ then $y \in F$, and

(2) if $x, y \in F$ then $x y \in F$.

If a proper filter is not contained in any other proper filter then it is called maximal; a proper filter is said to be prime if:

(3) Whenever $x_{1}+\cdots+x_{n}$ exists and is an element of $F$, then $x_{i} \in F$ for some $i \in\{1, \ldots, n\}$.

Let $L$ be a semilattice and $S \subseteq L$. The intersection of all filters in $L$ that contain $S$ is a filter $F$ and will be called the filter generated by $S$. It is easily seen that

$$
F=\left\{x \in L \mid x \geqq s_{1} \cdots s_{n} \text { for some } s_{1}, \ldots, s_{n} \in S\right\} .
$$

THEOREM 2.2. In a semilattice $L$, the following are equivalent:

(i) If $x_{1}+\cdots+x_{n}$ exists in $L$, then for each $x \in L, x x_{1}+\cdots+x x_{n}$ exists and equals $x\left(x_{1}+\cdots+x_{n}\right)$.

Received by the editors October 25, 1967. 
(ii) If $F$ is a filter in $L$ and $J$ a nonempty subset of $L$, disjoint with $F$, and such that $x_{1}+\cdots+x_{n}$ exists whenever $x_{1}, \ldots, x_{n} \in J$, then there exists a prime filter $F^{\prime}$ such that $F \subseteq F^{\prime}$ and $F^{\prime} \cap J=\varnothing$.

(iii) If $x$ then there exists a prime filter $F^{\prime}$ such that $x \in F^{\prime}$ and $y \notin F^{\prime}$.

Proof. (i) $\Rightarrow$ (ii). Let $\mathscr{P}$ be the set of all filters that contain $F$ and which are disjoint with $J$. Then $\mathscr{P}$ is partially ordered by inclusion and an application of Zorn's lemma shows that $\mathscr{P}$ has a maximal element $F^{\prime}$. Clearly $F^{\prime}$ is a proper illter, containing $F$, and disjoint with $J$. Suppose $x_{1}+\cdots+x_{n}$ exists and is an element of $F^{\prime}$ but $x_{i} \notin F^{\prime}$ for each $i \in\{1, \ldots, n\}$. Then for each $i$, let $F_{i}$ be the filter generated by $\left\{x_{i}\right\} \cup F^{\prime}$. By the maximality of $F^{\prime}, F_{i} \notin \mathscr{P}$ so $F^{\prime} \cap J \neq \varnothing$. Thus, for each $i$, there exists $j_{i} \in J$ such that $j_{i} \geqq x_{i} f_{i}$ for some $f_{i} \in F^{\prime}$. So for each $i$,

$$
f_{1} \cdots \cdots f_{n} \cdot x_{i} \leqq f_{i} x_{i} \leqq j_{i} \leqq j_{1}+\cdots+j_{n} .
$$

Since $x_{1}+\cdots+x_{n}$ exists, $\left[f_{1} \cdots f_{n} \cdot x_{1}\right]+\cdots+\left[f_{1} \cdots \cdot f_{n} \cdot x_{n}\right]$ exists and equals $f_{1} \cdots f_{n}\left(x_{1}+\cdots+x_{n}\right)$, which is in $F^{\prime}$. This implies that $j_{1}+\cdots+j_{n} \in F^{\prime} \cap J$, a contradiction. (ii) $\Rightarrow$ (iii). In (ii), let $F$ be the principal filter generated by $x$ and let $J=\{y\}$. (iii) $\Rightarrow$ (i). Clearly $x x_{i} \leqq x\left(x_{1}+\cdots+x_{n}\right)$ for each $i$. Suppose there exists $w \in L$ such that $x x_{i} \leqq w$ for all $i$, but $x\left(x_{1}+\cdots+x_{n}\right) \leq w$, then there exists a prime filter $F$ such that $x\left(x_{1}+\cdots+x_{n}\right) \in F$ and $w \notin F$. But then $x \in F$ and for some $i, x_{i} \in F$, which implies $w \in F$.

Definition 2.3. A semilattice in which one of the equivalent conditions in Theorem 2.2 holds will be called a prime semilattice.

Obviously a lattice is a prime semilattice if and only if it is distributive. Another example of a prime semilattice is obtained as follows. Let $X$ be an infinite set and let $L$ be the family of all subsets of $X$ whose set theoretic complements are infinite. $L$ is a prime semilattice under inclusion for if $x_{1}, \ldots, x_{n} \in L$ and $x_{1}+\cdots+x_{n}$ exists then it equals $x_{1} \cup \cdots \cup x_{n}$. Also see $\S 5$.

THEOREM 2.4. In a prime semilattice every maximal filter is prime.

Proof. Suppose that $F$ is a maximal filter in a prime semilattice $L$, that for some $n \geqq 2, x_{1}+\cdots+x_{n} \in F$, and that $x_{i} \notin F$ for $i=2, \ldots, n$. For each such $i$, the filter generated by $\left\{x_{i}\right\}$ and $F$ is not proper so $x_{1} \geqq x_{i} f_{i}$ for some $f_{i} \in F$. But then $f_{1} \cdots f_{n} \cdot x_{i} \leqq x_{1}$ for $i=2, \ldots, n$ so

$$
f_{1} \cdots f_{n}\left(x_{1}+\cdots+x_{n}\right)=\left[f_{1} \cdots \cdot f_{n} x_{1}\right]+\cdots+\left[f_{1} \cdots f_{n} x_{n}\right] \leqq x_{1} .
$$

Consequently, $x_{1} \in F$.

We adopt the following notation. If $L$ is a prime semilattice, $\mathscr{F}$ will be the family of all prime filters in $L$; for each $x \in L, x^{*}=\{F \in \mathscr{F} \mid x \in F\}$ and $L^{*}=$ $\left\{x^{*} \mid x \in L\right\}$. It is immediate from Theorem 2.2 (iii) that $L$ and $L^{*}$ are isomorphic under the correspondence $x \Leftrightarrow x^{*}$, where $L^{*}$ is partially ordered by inclusion. Furthermore,

(4) $\left(x_{1} \cdots \cdots x_{n}\right)^{*}=x_{1}^{*} \cap \cdots \cap x_{n}^{*}$ for all $x_{1}, \ldots, x_{n} \in L$. 
(5) $\left(x_{1}+\cdots+x_{n}\right)^{*}=x_{1}^{*} \cup \cdots \cup x_{n}^{*}$ whenever $x_{1}+\cdots+x_{n}$ exists in $L$.

Finally, let $R(L)$ be the smallest ring of subsets of $\mathscr{F}$ that contains $L^{*}$. By (4),

(6) $R(L)=\left\{x_{1}^{*} \cup \cdots \cup x_{n}^{*} \mid x_{1}, \ldots, x_{n} \in L\right\}$,

and from (5), we have

(7) $R(L)=L^{*} \cong L$ if and only if $L$ is a lattice.

DEFINITION 2.5. A semilattice $L$ is normally imbedded in a ring $R$ of sets if there exists an isomorphism of $L$ onto a subset of $R$ such that finite products in $L$ correspond to intersections in $R$ and finite sums in $L$-when they exist-correspond to unions in $R$.

THEOREM 2.6. A semilattice $L$ can be normally imbedded in a ring of sets if and only if $L$ is prime.

Proof. The above remarks show that the condition is sufficient. Conversely, suppose $\varphi$ is an isomorphism as defined above and suppose that $x_{1}+\cdots+x_{n}$ exists in $L$. If $x x_{i} \leqq w$ for $i=1, \ldots, n$ then

$$
\varphi\left(x\left(x_{1}+\cdots+x_{n}\right)\right)=\varphi(x) \cap\left(\varphi\left(x_{1}\right) \cup \cdots \cup \varphi\left(x_{n}\right)\right) \subseteq \varphi(w) .
$$

So $x\left(x_{1}+\cdots+x_{n}\right) \leqq w$.

3. The representation space of a prime semilattice. Let $L$ be a prime semilattice. We form a topological space by taking $L^{*}$ as a basis for a topology on $\mathscr{F}$. This space will be called the representation space of $L$ and will be denoted by $S(L)$. Since $R(L)$ is itself a distributive lattice, we can speak of the Stone space $S(R(L))$ of $R(L)$. Namely, $S(R(L))$ is the topological space consisting of the set $\mathscr{F}_{1}$ of all prime filters in $R(L)$, with $(R(L))^{*}=\left\{u^{*} \mid u \in R(L)\right\}$ as a basis for the topology, where $u^{*}=\left\{F_{1} \in \mathscr{F}_{1} \mid u \in F_{1}\right\}$ for each $u \in R(L)$. If $L$ is a distributive lattice then by (7), $S(L)$ is homeomorphic with $S(R(L))$; but we will show that this is the case even if $L$ is not a lattice. For this recall that a ring $R$ of subsets of a set $X$ is reduced provided that whenever $p, q$ are distinct elements of $X$, there exists $r \in R$ such that $R$ contains exactly one of $p, q$. Also, $R$ is perfect if for every prime filter $F_{1}$ in $R$ there exists an element $p \in X$ such that $F_{1}=\{r \in R \mid p \in r\}$.

LEMMA 3.1. If $L$ is a prime semilattice, then $R(L)$ is a reduced, perfect ring of subsets of $\mathscr{F}$.

Proof. If $F, F^{\prime}$ are distinct members of $\mathscr{F}$ and $x \in F-F^{\prime}$ then $F \in x^{*}, F^{\prime} \notin x^{*}$ and $x^{*} \in R(L)$. Now suppose $F_{1}$ is a prime filter in $R(L)$. Let $F=\left\{x \in L \mid x^{*} \in F_{1}\right\}$. Noting that $F_{1}$ is prime and consists of elements of the form $x_{1}^{*} \cup \cdots \cup x_{n}^{*}$ where $x_{i} \in L$, it is easily verified that $F$ is a prime filter in $L$ and that

$$
F_{1}=\left\{x_{1}^{*} \cup \cdots \cup x_{n}^{*} \in R(L) \mid F \in x_{1}^{*} \cup \cdots \cup x_{n}^{*}\right\} .
$$

THEOREM 3.2. If $L$ is a prime semilattice then $S(L)$ and $S(R(L))$ are homeomorphic.

Proof. For each $F \in S(L)$, let $\varphi(F)=\{u \in R(L) \mid F \in u\}$. Then $\varphi(F)$ is a prime filter and since $R(L)$ is reduced and perfect, $F \leftrightarrow \varphi(F)$ is a one to one correspondence 
between $\boldsymbol{S}(L)$ and $\boldsymbol{S}(R(L))$. To show $\varphi$ is a homeomorphism, it is sufficient to show $\varphi$ and $\varphi^{-1}$ preserve basic open sets. But this follows from

(8) $\varphi\left[x^{*}\right]=\left(x^{*}\right)^{*}$ and $\varphi^{-1}\left[\left(x^{*}\right)^{*}\right]=x^{*}$ for each $x \in L$.

To prove the first half of (8), let $F_{1} \in \varphi\left[x^{*}\right]$. Then $F_{1}=\varphi(F)$ for some $F \in x^{*}$ so $F_{1}=\{u \in R(L) \mid F \in u\}$. But $x^{*} \in F_{1}$ so $F_{1} \in\left(x^{*}\right)^{*}$. Now if $F_{1} \in\left(x^{*}\right)^{*}$ the $x^{*} \in F_{1}$. But $\varphi$ is onto, so there exists $F \in S(L)$ such that $\varphi(F)=F_{1}$. Therefore, $x^{*} \in \varphi(F)$ $=\{u \in R(L) \mid F \in u\}$. So $F \in x^{*}$ which means $F_{1} \in \varphi\left[x^{*}\right]$. The second half of (8) follows from the first since $\varphi$ is one-to-one.

The following properties of $\boldsymbol{S}(L)$ are immediate from Theorem 3.2. A c/o set is a set which is compact and open.

(9) $S(L)$ is a Stone space (i.e., $S(L)$ is a $T_{0}$ space such that the family of c/o sets is a basis and a ring of sets and such that if $\left\{u_{i} \mid i \in I\right\},\left\{v_{j} \mid j \in J\right\}$ are collections of c/o sets satisfying $\bigcap_{i \in I} u_{i} \subseteq \bigcup_{j \in J} v_{j}$ then there are finite sets $I_{1} \subseteq I, J_{1} \subseteq J$ such that $\bigcap_{i \in I_{1}} u_{i} \subseteq \bigcup_{j \in J_{1}} v_{j}$ ).

(10) $A$ is c/o in $S(L)$ if and only if $A \in R(L)$.

(11) $S(L)$ is compact if and only if there exists $x_{1}, \ldots, x_{n} \in L$ such that $F \cap$ $\left\{x_{1}, \ldots, x_{n}\right\} \neq \varnothing$ for each prime filter $F$ in $L$ (e.g., $S(L)$ is compact if $L$ has a greatest element).

In the Stone representation theory of distributive lattices, there is a one-to-one correspondence between the family of distributive lattices and the family of Stone spaces. Specifically, each distributive lattice is isomorphic with the family of $\mathrm{c} / \mathrm{o}$ sets of a Stone space and the c/o sets of any Stone space form a ring of sets. The situation for prime semilattices is described below.

Definition 3.3. A quasi-ring of sets is a collection $R$ of subsets of a set $X$ such that:

(12) If $r_{1}, r_{2} \in R$ then $r_{1} \cap r_{2} \in R$.

(13) If $r_{1}, \ldots, r_{n}, r \in R, r_{1} \cup \cdots \cup r_{n} \subseteq r$, and every element of $R$ that contains $r_{1} \cup \cdots \cup r_{n}$ also contains $r$, then $r=r_{1} \cup \cdots \cup r_{n}$.

THEOREM 3.4. Every prime semilattice $L$ is isomorphic with a quasi-ring $R$ of c/o sets of a Stone space $X ; L$ is a lattice if and only if $R$ consists of all $\mathrm{c} / \mathrm{o}$ sets of $X$. Conversely, every quasi-ring $R$ of c/o sets of a Stone space $X$ is a prime semilattice.

Proof. We have seen that $L \cong L^{*} \subseteq R(L)$ so by (10) and (7), the "only if" part of the proof will be complete if $L^{*}$ is a quasi-ring. Clearly (12) follows from (4). Suppose $x_{1}^{*}, \ldots, x_{n}^{*}, x^{*} \in L$. By the hypothesis of (13), and the fact that the correspondence $x \rightarrow x^{*}$ is an isomorphism, we obtain: $x_{1}+\cdots+x_{n}$ exists and equals $x$. So

$$
x^{*}=\left(x_{1}+\cdots+x_{n}\right)^{*}=x_{1}^{*} \cup \cdots \cup x_{n}^{*}
$$

Suppose now that $R$ is a quasi-ring of c/o sets of a Stone space $X$. Clearly $R$ is a semilattice with $r_{1} \cdot r_{2}=r_{1} \cap r_{2}$. If $r_{1}+\cdots+r_{n}$ exists, then by (12), $r_{1}+\cdots+r_{n}$ $=r_{1} \cup \cdots \cup r_{n}$ so $r r_{1}+\cdots+r r_{n}=r\left(r_{1}+\cdots+r_{n}\right)$ for each $r \in R$. 
4. The lattice of filters of a semilattice. Every algebraic (G. Birkhoff) or compactly generated (Dilworth and Crawley) lattice is isomorphic with the lattice of all filters of a meet semilattice. The lattice of all subalgebras of any abstract algebra with finitary operations (G. Birkhoff) is algebraic, and is isomorphic to the lattice of all ideals of the semilattice of finitely generated subalgebras. In view of these results, the next theorem might be useful.

THEOREM 4.1. Each of the following is a sufficient condition for a semilattice $L$, with a greatest element 1 , to be prime.

(i) The lattice of filters of $L$ is distributive.

(ii) Whenever $x y \leqq z(x, y, z \in L)$, there exists $x^{\prime}, y^{\prime} \in L$ such that $z=x^{\prime} y^{\prime}$ and $x \leqq x^{\prime}, y \leqq y^{\prime}$.

(iii) The lattice of all filters is a sublattice of the lattice of all subsemilattices.

Proof. (i) Suppose $x_{1}+\cdots+x_{n}$ exists and $x_{i} z \leqq w$ for each $i$. Then, denoting by $F_{a}$ the principal filter generated by $a$, we have $w \in \bigcap_{i}\left(F_{z}+F_{x_{i}}\right)=F_{z}+\bigcap_{i} F_{x_{i}}$. Since $x_{1}+\cdots+x_{n}$ exists, $\bigcap_{i} F_{x_{i}}=F_{x_{1}+\cdots+x_{n}}$ so that $w \geqq u v$ where $z \leqq u$ and $x_{1}+\cdots+x_{n} \leqq v$. Hence, $z\left(x_{1}+\cdots+x_{n}\right) \leqq u v \leqq w$, and $z\left(x_{1}+\cdots+x_{n}\right)=z x_{1}+\cdots$ $+z x_{n}$. (ii) Grätzer and Schmidt have shown that in a (join) semilattice, condition (ii) is equivalent to the distributivity of its lattice of ideals. The result then follows by (i) and duality. (iii) If $x y \leqq z$ then $z \in F_{x}+F_{y}$. By hypothesis $F_{x}+F_{y}=$ $\{u v \mid x \leqq u, y \leqq v\}$, so that $z=u v$ where $x \leqq u, y \leqq v$.

REMARK. None of these conditions are necessary for $L$ to be prime, as the following example shows. Let $L=\left\{0, x, y, z, a_{1}, a_{2}, \ldots\right\}$ where $0<x ; 0<z ; a_{1}>a_{2}$ $>a_{3}>\cdots>y>0 ; x<a_{i}, y<a_{i}$ for all $i$.

5. Implicative semilattices. An implicative semilattice is a semilattice $L$ satisfying:

(14) If $x, y \in L$ then there exists an element $x \rightarrow y$ such that for each $z \in L$, $x z \leqq y$ if and only if $z \leqq x \rightarrow y$.

Implicative semilattices are also known as relatively pseudo complemented semilattices. For the basic properties see [2, p. 141] and [5].

Pseudo complemented semilattices are not, in general, prime (e.g., the nonmodular lattice of five elements) but every implicative semilattice is prime. Indeed, if $x_{1}, \ldots, x_{n}, x$ are elements of such a semilattice and $x_{1}+\cdots+x_{n}$ exists then $x x_{i} \leqq x\left(x_{1}+\cdots+x_{n}\right)$ for each $i$. If $x x_{i} \leqq w$ for each $i$, then $x_{i} \leqq x \rightarrow w$ so $x_{1}+\cdots+x_{n}$ $\leqq x \rightarrow w$. Hence $x\left(x_{1}+\cdots+x_{n}\right) \leqq w$.

Every implicative semilattice contains a greatest element, denoted by 1 ; a least element, if it exists, will be denoted by 0 .

LEMMA 5.1. For an implicative semilattice $L$, with 0 , the following are equivalent:

(i) $F$ is a maximal filter in $L$.

(ii) $F$ is a prime filter in $L$ such that $(x+x \rightarrow 0) \in F$ for each $x \in L$. 
Proof. We have seen that every maximal filter is prime. Suppose $x \notin F$. Let $F_{1}$ be the filter generated by $\{x\} \cup F$. Then $F_{1}=L$ so $0 \geqq x f$ for some $f \in F$. Hence $f \leqq x \rightarrow 0$ so $x \rightarrow 0 \in F$. Conversely, suppose $F_{1}$ is a filter and $F \subset F_{1}$. Let $x \in F_{1}-F$. Since $x \notin F, x \rightarrow 0 \in F$, but then $0=x(x \rightarrow 0) \in F_{1}$ so $F_{1}=L$.

THEOREM 5.2. For an implicative semilattice $L$, with 0 , the following are equivalent :

(i) Every prime filter in $L$ is maximal.

(ii) $L$ is a Boolean algebra.

(iii) $S(L)$ is Hausdorff.

Proof. (i) $\Rightarrow$ (ii). It follows from Lemma 5.1 that

$$
x^{*} \cup y^{*}=([(x \rightarrow 0)(y \rightarrow 0)] \rightarrow 0)^{*} \text { for } x, y \in L .
$$

Thus, $L \cong L^{*}=R(L)$ is a distributive lattice. Now the dual lattice $D(L)$ is a distributive lattice with 0 and such that every prime ideal is maximal. By a theorem of Nachbin, $D(L)$ is a Boolean ring. But $D(L)$ has a greatest element so $D(L)$-and hence $L-$ is a Boolean algebra. (ii) $\Rightarrow$ (iii). Since $L$ is a Boolean algebra and $L \cong R(L) ; S(R(L))$ is Hausdorff. But $S(L)$ and $S(R(L))$ are homeomorphic. (iii) $\Rightarrow$ (i). Suppose $S(L)$ is Hausdorff, $F$ is a prime filter in $L$ and $x \notin F$. By Lemma 5.1, it is sufficient to show $x \rightarrow 0 \in F$. First, by (11), $S(L)$ is compact. Now $C\left(x^{*}\right)$-the set theoretic complement of $x^{*}$-is a closed set in a compact Hausdorff space. Hence $C\left(x^{*}\right)$ is a c/o set. By $(10), C\left(x^{*}\right)=x_{1}^{*} \cup \cdots \cup x_{n}^{*}$.

Assertion: $x_{1}+\cdots+x_{n}$ exists and equals $x \rightarrow 0$. To see this, note that for each $i$, $x_{i}^{*} \subseteq C\left(x^{*}\right)$ so $\left(x x_{i}\right)^{*}=x^{*} \cap x_{i}^{*}=\varnothing=0^{*}$. Thus $x x_{i}=0$ and $x_{i} \leqq x \rightarrow 0$. If $x_{i} \leqq w$ for $i=1, \ldots, n$ but $x \rightarrow 0 \$ w$ then there exists a prime filter $F^{\prime}$ such that $x \rightarrow 0 \in F^{\prime}$ but $w \notin F^{\prime}$. Then $x \notin F^{\prime}$ so $F^{\prime} \in C\left(x^{*}\right)$. Consequently, $F^{\prime} \in x_{i}^{*}$ for some $i$, so $x_{i} \leqq w$ and $x_{i} \in F^{\prime}$ imply the contradiction $w \in F^{\prime}$.

Now the assertion means that $C\left(x^{*}\right)=(x \rightarrow 0)^{*}$ and so $x \notin F$ implies $x \rightarrow 0 \in F$.

Finally we have the analogue of Theorem 3.4 .

THEOREM 5.3. Every implicative semilattice $L$ is isomorphic with a quasi-ring $R$ of c/o sets of a Stone space $X$ such that

(15) If $r_{1}, r_{2} \in R$ then int $\left(C\left(r_{1}\right) \cup r_{2}\right) \in R$.

Furthermore, $R$ is a subimplicative-semilattice of the implicative semilattice of open sets of $X$. Conversely, every quasi-ring of $\mathrm{c} / \mathrm{o}$ sets of a Stone space satisfying (15) is an implicative semilattice.

Proof. Let $L$ be an implicative semilattice. Then $L \cong L^{*}$ is a quasi-ring of c/o sets of $S(L)$. If $x^{*}, y^{*} \in L^{*}$ and $F$ is a prime filter then $(x \rightarrow y) \in F$ implies $x \notin F$ or $y \in F$ so $(x \rightarrow y)^{*} \subseteq C\left(x^{*}\right) \cup y^{*}$. But $(x \rightarrow y)^{*}$ is the largest open set contained in $C\left(x^{*}\right) \cup y^{*}$, for if $\cup_{i} x_{i}^{*} \subseteq C\left(x^{*}\right) \cup y^{*}$, then $x_{i}^{*} \subseteq C\left(x^{*}\right) \cup y^{*}$ so $x x_{i} \leqq y$ for all $i$. Hence $x_{i} \leqq x \rightarrow y$ for each $i$ and so $\bigcup_{i} x_{i}^{*} \subseteq(x \rightarrow y)^{*}$. This shows that

$$
\text { int }\left(C\left(x^{*}\right) \cup y^{*}\right)=(x \rightarrow y)^{*}
$$

and hence (15). Also in the implicative lattice $L^{\prime}$ of open sets of $X$, we have $x^{*} \rightarrow y^{*}$ 
$=\operatorname{int}\left(C\left(x^{*}\right) \cup y^{*}\right)=(x \rightarrow y)^{*}$ so that $R$ is a subimplicative-semilattice of $L^{\prime}$. The remainder of the theorem follows from Theorem 2.4 and (15).

I wish to thank the referee for his comments and suggestions concerning this paper.

\section{REFERENCES}

1. Garrett Birkhoff, Lattice theory, 3rd ed., Amer. Math. Soc. Colloq. Publ., Vol. 25, Amer. Math. Soc., Providence, R. I., 1967.

2. H. B. Curry, Foundations of mathematical logic, McGraw-Hill, New York, 1963.

3. O. Frink, Pseudo-complements in a semi-lattice, Duke Math. J. 29 (1962), 505-514.

4. A. Horn, Notes on Boolean algebras, (unpublished).

5. W. Nemitz, Implicative semi-lattices, Trans. Amer. Math. Soc. 117 (1965), 128-142.

6. H. Rasiowa and R. Sikorski, The mathematics of metamathematics, Monogr. Mat., Tom 41, Polska Akademia Nauk, Warsaw, 1963.

7. E. T. Schmidt, Über die Kongruenzverbände der Verbände, Publ. Math. Debrecen 9 (1962), 243-256.

8. M. H. Stone, Topological representations of distributive lattices and Browerian logics, Căsopis Pěst. Mat. Fyz. 67 (1937), 1-25.

UNIVERSity of CALIFORNiA, Los ANgeles, California 